\title{
Interfaces
}

INTERFACES Image Texte Language

$36 \mid 2015$

Synaesthesia

\section{De la synesthésie : The Foundations of Aesthetics} (1922)

\section{Bénédicte Coste}

\section{(2) OpenEdition}

1 Journals

\section{Édition électronique}

URL : http://journals.openedition.org/interfaces/233

DOI : 10.4000/interfaces.233

ISSN : 2647-6754

Éditeur :

Université de Bourgogne, Université de Paris, College of the Holy Cross

\section{Édition imprimée}

Date de publication : 1 janvier 2015

Pagination : 95-112

ISBN : 9780983175247

ISSN : $1164-6225$

\section{Référence électronique}

Bénédicte Coste, «De la synesthésie : The Foundations of Aesthetics (1922) », Interfaces [En ligne], 36 | 2015, mis en ligne le 01 janvier 2018, consulté le 04 janvier 2021. URL : http://

journals.openedition.org/interfaces/233; DOI : https://doi.org/10.4000/interfaces.233

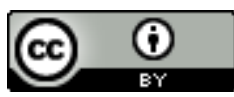

Les contenus de la revue Interfaces sont mis à disposition selon les termes de la Licence Creative Commons Attribution 4.0 International. 


\section{DE LA SYNESTHÉSIE : THE FOUNDATIONS OF AESTHETICS (1922)}

\section{Bénédicte Coste}

Rares sont les commentateurs qui s'intéressent aujourd'hui à The Foundations of Aesthetics ${ }^{1}$. Bien qu'il figure dans les œuvres complètes d'Ivor Armstrong Richards, ce court ouvrage est le résultat d'un travail à plusieurs mains - Richards, Charles K. Ogden et l'historien de l'art et peintre James Wood - entamé en 1920 à partir d'un intérêt commun pour l'analyse du langage de la critique d'art ${ }^{2}$. En dépit de son titre pompeux, l'une des " covert jokes $»^{3}$ dont C. K. Ogden était familier, il se veut recherche d'une nouvelle façon d'évaluer l'art en invitant son lecteur à lier le(s) texte(s) et l'image à travers une singulière esthétique du livre. Ses auteurs réévaluent les idées dominantes, issues de la réflexion sur le beau inaugurée au XVIII siècle par Hutcheson, Burke, Baumgarten et poursuivie par Kant, Schiller, les esthéticiens romantiques et victoriens, et les modernistes : Vernon Lee, Roger Fry, et Clive Bell. Plus précisément, les trois auteurs rejettent l'idée d'un état ou d'une émotion esthétique spécifique et cherchent à étudier et à apprécier la beauté à travers les arts et l'expérience quotidienne (71).

The Foundations introduit la technique de la « définition multiple » d'un terme qui sera utilisée dans les ouvrages suivants de Richards. Le terme « beauté » reçoit ainsi 16 définitions (« The Senses of Beauty ») dont 15 sont rejetées ou déclarées insuffisantes ou confuses avant que les auteurs proposent leur propre théorie relevant de ce qu'ils appellent « synesthésie », laquelle met en jeu les catégories d'harmonie et d'équilibre, et dont ils trouvent une élaboration chez Confucius.

La définition de la synesthésie promue par ce traité mérite donc d'être explicitée. Si la synesthésie est aujourd'hui définie comme un phénomène psycho-neurologique consistant en un état intensifié de plusieurs sens répondant simultanément à une expérience, les trois auteurs en offrent une

1 I. A. Richards, C.K. Ogden, James Wood, The Foundations of Aesthetics, 1925, éd. John Constable, London and New York: Routledge, 2001. Toutes les références renvoient à cette édition. Toutes les traductions sont de l'auteur. L'édition de Constable cite 8 recensions entre 1922 et 1948.

2 Les premiers articles de Richards portent sur l'esthétique : « Art and Science », « Emotion and Art », « Four Fermented Aesthetics », «The Instruments of Criticism : Expression ». Dans une lettre à sa future épouse, Richard écrira qu'il cherche à « mettre les arts au rang de mode suprême de communication, au premier plan de toutes les valeurs », Lettre au Dr Pilley, 19 novembre 1923, The Selected Letters of I. A. Richards, éd. J. Constable, Oxford: Clarendon, 1990, p. 27.

3 The Foundations of Aesthetics, p. 1viii. 
définition différente pour donner une « nouvelle orientation » aux théories esthétiques (2). Présentant plusieurs d'entre elles pour montrer la « sphère de validité de chacune » (2), ils précisent que leur nombre contribue à l'appréciation de l'art. La théorie de la synesthésie clôture l'ouvrage, s'imposant comme principe global, unification des tendances centrifuges développées par les autres esthétiques, Grund sur lequel elles ont joué. Plus approfondie, elle vise à clarifier les confusions nées des diverses définitions, angles d'approche de la beauté et des différentes facettes à travers lesquelles s'aborde la relation artistique (l'objet, l'effet, le rapport du beau à la nature et au génie). Elle se définit dès 1922 comme « une explication des expériences esthétiques décrites par nombre des artistes et des critiques les plus grands et les plus sensibles du passé, [et qui] pourrait être considérée comme la théorie de la Beauté par excellence $»^{4}$.

Ce qui suit sera donc consacré à présenter la synesthésie, puis les procédés avec lesquels les auteurs invitent le lecteur à en faire l'expérience à travers la composition matérielle de l'ouvrage. Sa version originale, "The Sense of Beauty », était dépourvue d'illustration", avant la première édition parue chez G. Unwin en 1922, édition elle-même révisée en 1925 et abondamment illustrée.

The Foundations est un traité inhabituel, et d'une certaine complexité matérielle. Très court, il s'accompagne de 16 reproductions photographiques d'œuvres d'art de différentes époques ${ }^{6}$, certaines d'origine européenne, d'autres d'Afrique, d'Inde et de Chine, la « Préface » originale soulignant que l'intérêt pour l'esthétique s'est renforcé via la connaissance de l'art extra-européen ${ }^{7}$. Spécialement créées pour l'occasion, ces reproductions sont destinées à illustrer les thèses examinées auxquelles elles renvoient explicitement ${ }^{8}$. Elles voisinent également avec des citations littéraires plus ou moins connues, quoique non référencées, dans le corps du texte, fonctionnant comme « illustration concrète de certains points de la discussion du passage qui vient immédiatement avant $»$ et "rappel ${ }^{9}$ de la discussion en cours. Elles sont en outre destinées à distraire le lecteur de l'utilisation d'un langage trop

4 «Préface à l'édition de 1922 », p. 80-1.

5 Cambridge Magazine, 10.2 (Jan-Mar 1921), p. 73-93. Le texte sera utilisé dans le chapitre VII de The Meaning of Meaning.

6 La plupart se trouvent au British Museum.

7 « non-European », « Préface à l'édition de $1922 »(80)$.

8 «the reproductions, most of which have been specially made for the purpose. [...] Each, [...] adequately illustrates one or more of the theories discussed, and it will be obvious that all of them are works of high rank. » (81)

9 « Préface originale », p. 3. 


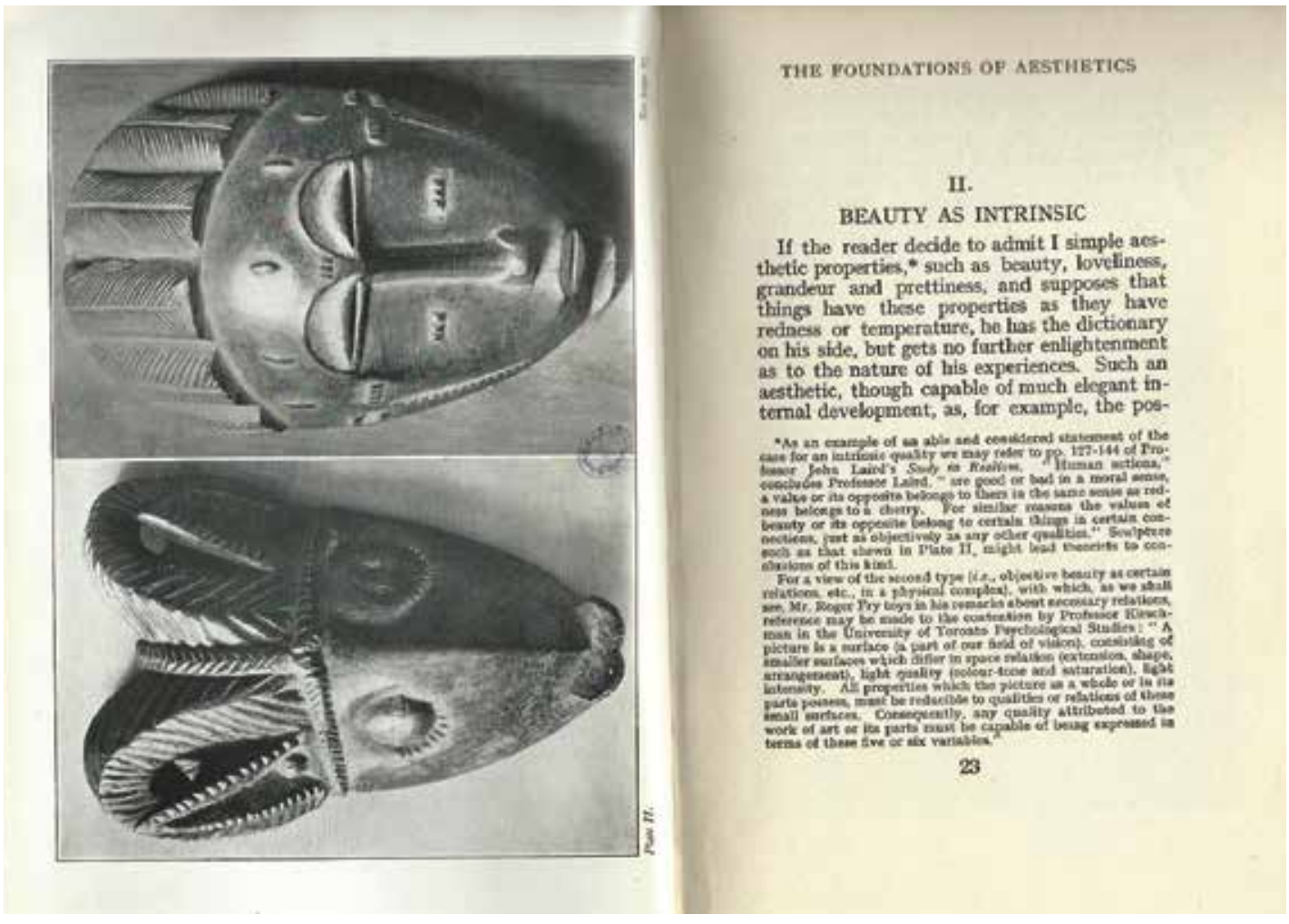

Fig. 1: Planche II et page 23 de OGDEN, C. K., I. A. RICHARDS, and James WOOD. The Foundations of Aesthetics. London: Allen, 1925. 
scientifique (81). Leur titre apparaît en fin d'ouvrage, dans un index qui fait suite à l'index nominorum. Le texte déjà éclaté entre prose, poésie et image se divise également entre le corps du texte et les notes parfois très abondantes (Fig 1). Typographiquement, ce petit traité est un « eye-twister » et un «mindboggler $»^{10}$ invitant son lecteur à regarder ("see ») l'image au détour d'une note infrapaginale, ou à revenir en arrière, poursuivant la discussion dans les notes parfois plus importantes que le corps du texte - c'est le cas du chapitre VIII -, fournissant plusieurs paratextes (index, listes, préfaces) qui font dérailler la lecture linéaire traditionnelle et mettent le/s texte/s, les images et les paratextes en tension.

La première édition s'ouvrait sur une Préface, basculée en 1925 en fin d'ouvrage, à la suite des index ${ }^{11}$. La liste des illustrations visuelles apparaît quant à elle, au début de l'ouvrage, à la suite du Frontispice montrant une statue féminine grecque primitive. Enfin, un idéogramme chinois et une citation du Zhong Yong (la doctrine du Milieu ${ }^{12}$ ) choisie par Wood, ornent l'ouvrage (Fig 2, p. 13).

Il convient donc de ne pas se laisser abuser par l'utilisation du terme « illustration » et de voir dans cette disposition typographique et dans ces œuvres des « stimuli », au sens richardsien, destinés à l'éducation artistique qu'il jugeait indispensable aux « lecteurs lambda ${ }^{13}$. La juxtaposition du texte et de l'image fonctionne comme une mise en actes de la théorie de la synesthésie avancée ici. Par le jeu du textuel et du visuel, par l'utilisation de la composition paginale, par la concision de l'écriture, The Foundations invite son lecteur à une lecture déroutante, en zigzag, placée sous le signe du déséquilibre typographique et de la tension visuelle. Si l'on considère que la synesthésie résulte de l'harmonisation et de l'équilibre des impulsions, cet ouvrage est à lire comme proposition synesthétique.

10 " In some cases neither the illustrations nor the quotations seem happily chosen: at least I have found it difficult to see what theory was specially exemplified by some of the illustrations. And some well-known passages would afford better examples of points under discussion than several of those given » avouera $\mathrm{C}$. W. Valentine dans sa recension de The Foundations, Mind, ns, 32.125 (Jan., 1923), p. 120.

11 L'édition de J. Constable a augmenté cette complexité.

12 C'est le premier des Quatre livres, fondements du confucianisme, par Zi Si sous la dynastie Zhou. Il s'agit de courts textes attribués à Confucius et de neuf chapitres de commentaires par Zeng Zi.

13 I. A. Richards, " Chapter VII. A Psychological Theory of Value », The Principles of Literary Critcism (1924), London: Routledge, 1989, p. 33-42, où est développée la théorie psychologique (largement behaviouriste) et sont définis les stimuli, les impulsions, les émotions et les attitudes. 


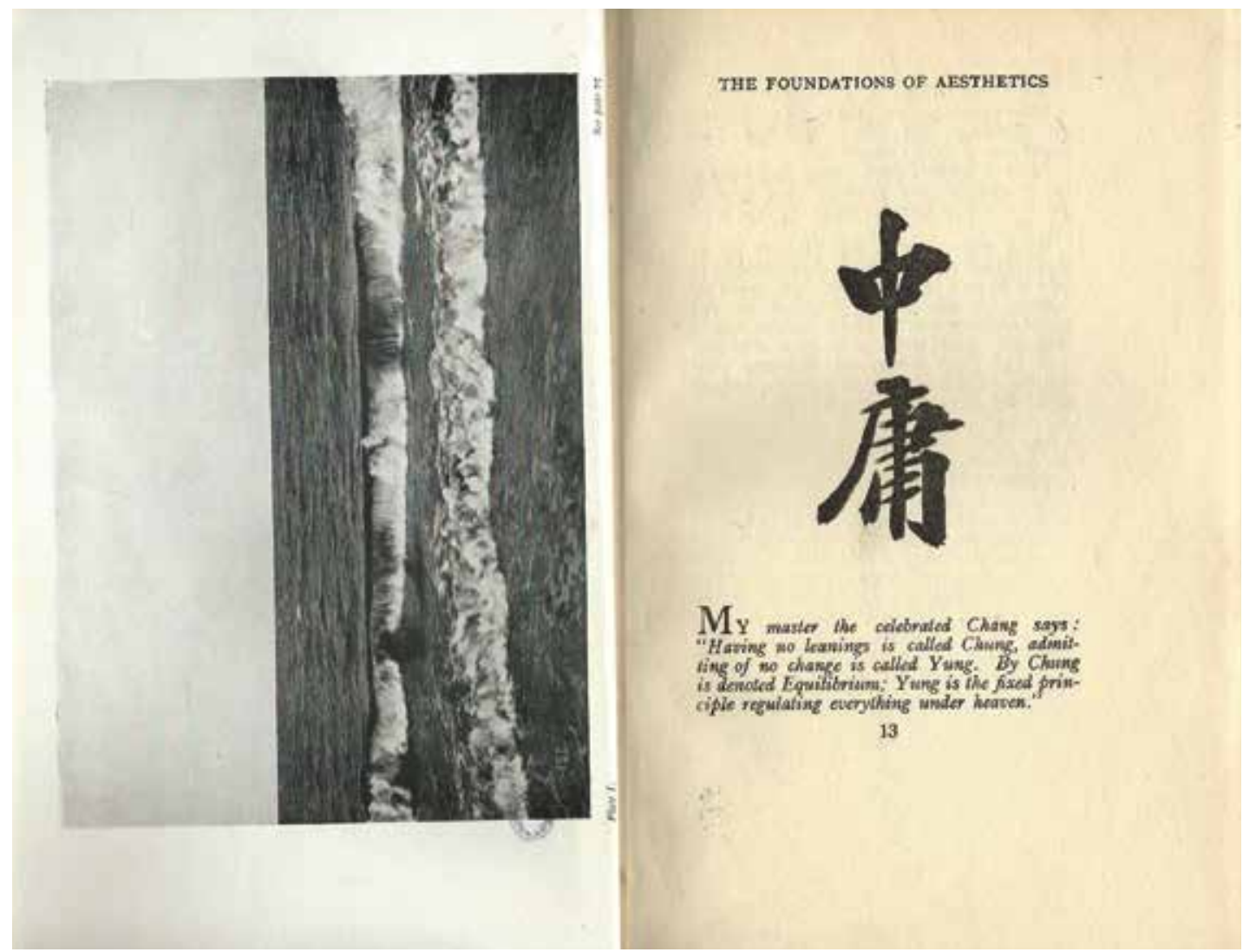

Fig. 2: Planche I et page 13 de OGDEN, C. K., I. A. RICHARDS, and James WOOD. The Foundations of Aesthetics. London: Allen, 1925. 


\section{Qu'est-ce que la synesthésie?}

La définition de la synesthésie s'appuie sur une phénoménologie de l'expérience. Nombre de poètes et de critiques ont en effet repéré une expérience qui « délimite un champ ne pouvant être défini autrement et expliquant également pourquoi les objets qu'il contient peuvent raisonnablement être considérés comme possédant une grande importance»(62). Ses limites ne sont pas celles de la « beauté »; elle est « fugace et évanescente à l'extrême»(62). C'est par exemple, dans le champ visuel, notre conscience de certaines formes et de certaines couleurs qui se révèlent comme telles :

À travers ce processus, des impulsions [psycho-physiologiques] sont suscitées et soutenues, lesquelles voient leur variété et leur degré de systématisation augmenter. Aux stages initiaux de ces systématisations correspondront des émotions comme la joie, l'horreur, la colère et la joie, ou des attitudes comme l'amour, la vénération, la sentimentalité. (63)

Ces émotions sont celles du spectateur et partiellement celles du créateur. Elles fournissent ses conditions de possibilité à la relation esthétique entendue au sens étymologique du grec aisthesis désignant la capacité de sentir, non pas individuellement mais avec autrui (syn). Si l'esthétique des trois auteurs prend l'individu comme unité minimale, elle pose cependant la question de la relation au tiers, au groupe, et d'abord à l'artiste.

Que met de lui-même un artiste dans son œuvre ? Une « sélection » et un « arrangement » qui proviennent de « la direction et de l'accentuation de son intérêt [...] pour le jeu de ses impulsions qui contrôlent son activité à ce moment, et il se trouve que c'est souvent le même groupe d'impulsions qui est suscité chez le spectateur»(63). L'artiste ne diffère pas du « contemplateur » : il connaît des impulsions dont la systématisation contribue à façonner son œuvre. Ce contact entre artiste et " contemplateur » est une question de degré. À ce stade, nous n'avons pas fait l'expérience de la beauté, mais notre « émotion prend un certain caractère général, et nous découvrons que notre attitude est devenue en retour impersonnelle»(63). Le dernier terme est à entendre comme libération des préjugés et des représentations gouvernant notre expérience quotidienne et entravant notre accès à la beauté.

Lorsque « les impulsions variées [...] sont devenues encore plus systématisées et intensifiées » (64), nous pouvons faire l'expérience de la synesthésie en prélude à l'expérience de la beauté. Dans cette perspective, « une systématisation complète doit prendre la forme d'un ajustement qui préservera le libre jeu de chaque impulsion tout en évitant totalement la frustration. » Et c'est « [e]n tout équilibre de cette sorte, même momentané [...que] nous faisons l'expérience de la beauté»(64). Cet état psychologique où les impulsions sont expérimentées de concert quoique sans confusion est la synesthésie, définie 
comme équilibre et harmonie (64). L'harmonie s'opère via le concert des impulsions ; l'équilibre obère l'action sans pour autant que le sujet puisse être qualifié de passif ${ }^{14}$.

L'effet de l'expérience synesthétique de la beauté consiste à nous rendre à nous-mêmes, c'està-dire paradoxalement à un état d'impersonnalité lié au libre jeu et à l'harmonisation des impulsions : «En prenant conscience de la beauté, nous devenons plus entièrement nous-mêmes au fur et à mesure que nos impulsions sont engagées (lorsque notre intérêt n'est pas canalisé dans une direction, il y a détachement). Nous devenons impersonnels ou désintéressés»(65-6). Cette libération est héritée du désintéressement arnoldien opposé à l'esprit sectaire et aux propos extra-littéraires qui entravaient, selon Matthew Arnold, le jugement poétique de ses contemporains ${ }^{15}$. Les trois auteurs actualisent l'exigence de leur prédécesseur et la dépassent : plus nous nous différencions des individualités ou des objets autour de nous, en devenant ainsi nous-mêmes, plus ces derniers acquièrent leur ipséité. Et le « corollaire de cette individualisation est que des groupes d'impulsions sont ressentis en relation avec d'autres»(66-67). L'art a donc un pouvoir de différenciation subjective dont le spectateur fait l'exercice. Se découvrant par différenciation d'autrui ou des objets, il permet leur compréhension. La relation esthétique envisagée par le truchement de l'œuvre conduit implicitement à une éthique. Dans cette perspective, l'éducation consiste à reconnaître la valeur de l'art dérivant de ce « pouvoir intensifié de différenciation » (3) ${ }^{16}$. L'art devient « un moyen d'établir des relations avec des personnalités qui ne seraient pas autrement accessibles » (67). Il est un opérateur d'altérité, et il l'est d'autant plus qu'il nous rend étrangers à nous-mêmes en nous purifiant mentalement dans l'expérience synesthétique.

La gamme des impulsions impliquées dans l'expérience de la beauté explique l'intensification, l'élargissement ou l'approfondissement de ce que les trois auteurs appellent leur coloration. L'art n'est cependant pas le seul à susciter une semblable gamme d'impulsions et à les harmoniser. Nous en faisons l'expérience « à travers des circonstances très différentes et en rapport avec des objets habituellement

14 Une œuvre d'art ne devrait pas nous pousser à agir, sauf à devenir alors « stimulante» (65), axiome constant chez Richards, qui lui servira à promouvoir la valeur de la poésie.

15 M. Arnold, «The Function of Criticism at the Present Times » (1864) : « how is criticism to show disinterestedness? By keeping aloof from what is called "the practical view of things"; by resolutely following the law of its own nature, which is to be a free play of the mind on all subjects which it touches. By steadily refusing to lend itself to any of those ulterior, political, practical considerations about ideas, [...] which criticism has really nothing to do with » (Lectures and Essays in Criticism, p. 35).

16 Ce pouvoir n'est pas sans évoquer l'éducation esthétique selon W. Pater : « Our education becomes complete in proportion as our susceptibility to these impressions increases in depth and variety. » "Preface», The Renaissance. Studies in Art and Poetry (1873), éd. Adam Philips, Oxford: Oxford Classics, 1988, p. xxx. 
considérés comme ne relevant pas de l'art » (69). C'est par exemple le cas des opérations scientifiques ${ }^{17}$. Distinct de la science, l'art est expérimenté par un sujet néanmoins capable d'unité psychique par-delà la différence de ses expériences. Il n'existe donc pas d'état esthétique distinct ${ }^{18}$.

\section{Différentes expériences de lecture, de texte et d'image}

À cette unité subjective, née du libre jeu des impulsions, s'oppose en apparence la facture de l'ouvrage mêlant poésie, prose et image pour examiner des théories déclarées insatisfaisantes. Leur classement participe d'une volonté philosophique, mais leur présentation contribue également aux aspérités visuelles et cognitives dont se hérisse ce petit traité. L'ouvrage est une praxis ; il met en tension texte /s et image/s, dans une volonté paradoxale d'harmonie et d'équilibre qui s'atteint in fine. Il nécessite donc un commentaire linéaire, quoique synthétique.

Selon les auteurs, les théories esthétiques peuvent logiquement se ranger en trois catégories, A, B, C. La catégorie A inclut les théories de la beauté relevant de la tautologie ou de la forme spécifique (chapitres I et II). La catégorie B inclut les théories de l'imitation (de la mimesis) et celles qui soulignent son effet social (chapitres III à VIII). La catégorie C inclut les théories de la beauté comme expression, cause de plaisir ou d'émotion(s), mise en jeu du processus d'empathie (l'Einfülhung), et enfin, last but not least, la synesthésie (chapitres IX à XVI).

Cette catégorisation, n'obéissant pas à l'histoire de l'esthétique, renvoie à des théories soutenues par des philosophes dont certains sont cités dans les notes en compagnie d'autres aujourd'hui moins connus et qui témoigne d'une connaissance approfondie de la réflexion au début du siècle. Elle dessine également une ligne de fracture entre les critiques du groupe de Bloomsbury et les Cambridgiens concernant la question de l'engagement social des écrivains ${ }^{19}$. La différence la plus notable se situe toutefois entre l'esthétique qu'ils revendiquent et l'esthétique allemande ou anglaise qu'ils souhaitent dépasser en examinant la question de la beauté à nouveaux frais.

17 Se dessine la distinction du langage poétique et du langage scientifique qui sera faite dans The Principles of Literary Criticism et dans Science and Poetry (1926). Richards introduit le terme « pseudo-statement » pour décrire des énoncés émotifs ou poétiques et extraire les seconds du registre de la vérité scientifique.

18 Dans The Principles of Literary Criticism (1924), Richards consacre un chapitre à railler « The Phantom of the Aesthetic State », The Principles of Literary Criticism, p. 6-11.

19 Voir Joshua Kavaloski, «The New Critics and the Social Function of Modern Literature », High Modernism: Aestheticism and Performativity in Literature of the 1920's, London: Camden House, Inc, 2014, p. 39-72; et Benjamin Kohlmann, « Responsible Propagandists » I.A. Richards, T. S. Eliot and Cambridge Experiment », Committed Styles: Modernism, Politics, and Left-Wing Literature in the 1930s, Oxford: OUP, 2014, p. 18-53. 
Résumées avec une grande concision, les théories sont accompagnées des " illustrations » décrites plus haut dont la valeur est polyvalente : elles peuvent être de l'ordre d'un accompagnement, d'une scansion, ou renforcer les commentaires ironiques auxquels se livrent les auteurs. Cette valeur n'est jamais neutre et invite le lecteur à un travail interprétatif constituant justement les prémisses de la valeur éducative attribuée à l'art. L'illustration apparaît donc comme un stimulus visuel dont se révèle la valeur d'altérité et donc d'altérisation et d'ipséisation du sujet. Ainsi, les deux masques africains (reproduction B) viennent-ils conclure le premier chapitre, "Expériences esthétiques », rappelant l'existence de diverses définitions. Nous sommes ici regardés par ces masques sans pupille qui évacuent toute idée préconçue de la beauté. Une tête en bois africaine presque dépourvue de traits et de sexe (reproduction C) ouvre le chapitre II, « La beauté intrinsèque », soulignant l'impossibilité de définir le beau à partir de ses propriétés esthétiques, puisqu'il cesse d'être analysable et devient affaire de préférence personnelle. Ces deux reproductions renvoient également à la Préface mettant en exergue la dette des auteurs à l'égard de l'art extra-occidental dont la valeur inattendue va progressivement apparaître.

Le chapitre III, «L'imitation », s'orne du portrait de la Dame à l'hermine, actuellement attribué à Léonard (reproduction D). Illustration de la mimesis aristotélicienne liant plaisir et reconnaissance du modèle exemplairement signifiée par ce portrait ? Ou bien nostalgie devant une peinture représentative que la configuration religieuse et la technologie actuelle - ayant dévalorisé toute idée de l'image comme réplique de la création divine - ont relégué au rang d'art dépassé, si l'on en croit nos auteurs (19-20) ? Quelques vers d' «Ariel to Miranda » de Shelley (19) résument la théorie d'un art mimétique mais introduisent également une nuance ironique pour dénoncer la confusion du jugement personnel et de la beauté par les amateurs de banalités.

Le chapitre IV, « Le médium », s'ouvre par la reproduction d'une gigantesque statue d'une épouse de Ramsès II (reproduction E), que le lecteur est invité à confronter aux théories du beau comme rapport au médium. Ces dernières sont séparables, en deux tendances : l'une fait de l'art l'exploitation habile du médium adéquat, et l'autre souligne la nécessité de son ajustement au sujet. Mais, ajoutent les auteurs, si l'on réduit l'art à son médium, chaque art possède un matériau produisant un effet distinct sur nos impulsions, et donc sur nos sentiments. En outre, si l'artiste reproduit ce qui l'a affecté, il faut donc qu'il ait vécu et que l'art et l'existence ne soient pas séparés, sauf à vouloir réduire « le champ de l'expérience esthétique » (25). En témoignent quatre vers du « Queen Mab » de Shelley, invitant au dépassement des habitudes mentales $(24)^{20}$, et le Journal de Delacroix, cité en note, louant le charme des vivants plutôt que celui des œuvres. S'il est impossible de supprimer ce qui relève de l'expérience

20 Le quatrain offre une autre vision de la mort... 
réelle, le spectateur doit réagir en mettant en jeu des impulsions similaires à celle de l'artiste à partir de sa propre expérience (25-6), et il est donc nécessaire qu'il ait également vécu.

D'autre part, le contenu représentatif de l'art est suffisamment important pour être préservé. L'Auld Lang Syne de Burns (26) apparaît alors comme une pointe ironique adressée aux partisans d'un art non-représentatif, avant qu'une citation de Lafcadio Hearn renforce l'exigence de ne pas négliger la persistance de la représentation ainsi que l'influence des expériences passées. À ce que Lafcadio Hearn nomme la " mémoire organique » (26) correspondent les " Princes de la maison de Timour dans un pavillon ", de l'école mongole du XVII ${ }^{\mathrm{e}}$ siècle (reproduction F). Servant de support à toute expérience dont celle de la beauté (26), la mémoire organique se trouve ainsi présentée sous les aspects de l'altérité géographique et culturelle, pour désigner son oubli, peut-être au profit d'un présentéisme malheureux.

Nulle reproduction ne semble illustrer le chapitre « Le génie » (28). Ce chapitre se compose de deux courts paragraphes réussissant par leur brièveté et leur référence à des essayistes mineurs à réduire à néant la théorie d'un génie forcément individuel ${ }^{21}$. Le parallèle entre un art compris comme le produit d'un génie et la vigne comme une production de Madère constitue une pointe ironique consonnant avec la brièveté de la réfutation. Par un contraste particulièrement marqué sur le plan visuel, une longue note ${ }^{22}$ montre qu'il s'agit en outre d'une définition élitiste et solipsiste de l'art, quiconque pouvant s'arroger le droit d'appartenir à la catégorie des génies. L'ironie se renforce du distique « Naebody cares for me, / I care for nobody » concluant « I hae a wife » de Burns, pour illustrer cette position solipsiste (28). L'idée d'un génie contrevient donc à la définition de la beauté et du sujet donnée ici. Le génie individuel ne saurait exister, sauf à établir une différence, et à interdire justement l'expérience de la beauté à certains individus.

Le chapitre «La vérité » accentue la veine ironique des auteurs qualifiant les théories de la vérité en art ou de l'art comme expression de la vérité de doctrines « révélatoires » (30), relevant du « mysticisme » par ailleurs prêté à M. Arnold et Coleridge (31). Car si l'on suit Coleridge pour qui l'émotion suscitée par une œuvre symbolise la vérité de l'expérience individuelle passée, l'art devient une échappatoire, thèse apparentée à son idéalisation. Il n'est donc pas étonnant que le ton faussement blasé du dernier chant (IV), du Childe Harold de Byron (33) conclue cette analyse ironique. Car si l'art devient un moyen d'évasion, il perd sa valeur éducative et politique.

21 Les auteurs citent les écrits de Külpe (1907) et la littérature d'inspiration bergsonienne. Le chapitre VII, "L'Illusion », se réduit pareillement à deux paragraphes caustiques. Faire de l'illusion la fin de l'art, c'est retomber dans un art de l'évasion supérieur à la réalité. Réduire l'art à une évasion addictive conduit au désintérêt du public et à la dévaluation de l'art.

22 Les auteurs citent Henri Bergson: an Account of his Life and Philosophy de Ruge. 
The March to Finchley d'Hogarth (reproduction G) sert de transition au chapitre « Les effets sociaux ", examinant l'utilité sociale prêtée à l'art par un grand nombre de penseurs et d'essayistes dits idéalistes, de J. Ruskin à J. Middleton Murry en passant par W. Morris. Ces « doctrines de l'élévation sorties des foyers laborieux des moralistes de la fin de l'époque victorienne » (35) s'opposent à «l'attitude aristocratique » raillée de nouveau par un extrait du chant IV du Childe Harold de Byron (36), ce qui laisse supposer que les moralistes et les aristocrates d'opérette ne sont peut-être pas si différents. La fonction utilitaire prêtée à l'art s'inscrit en outre, selon les auteurs, dans le cadre de la résurgence des bons sentiments liés à l'immédiat après-guerre, sentiments dont ils décèlent l'origine protestante (36). Une note renvoie aux Curiosités esthétiques de Baudelaire (1868) qui déplorait déjà « la prétention philosophique, mélancolique et raphaélesque » des " petits parias » peints par Millet désireux d'ajouter une dimension sociale à son art (36). Depuis les années 1860 - époque des valeurs mid-victoriennes rejetées par les modernistes dans les années 1920 - n'y a-t-il pas eu de changement dans la valeur assignée à l'art ?

Dans le chapitre suivant, «L'expressionnisme », l'ironie de cette question toute rhétorique s'exerce plus amplement contre B. Croce dont l'idéalisme métaphysique s'appuie sur le langage spéculatif pour énoncer l'évidence, tandis que ses partisans britanniques (Carritt et Eddington) se voient renvoyés à l'obscurité d'une note.

Aux côtés de théories remontant à l'évolutionnisme scientifique des années 1870, la théorie spencérienne de l'art comme jeu et comme méthode d'accroissement de l'énergie libre (41) conduit quant à elle à contempler les œuvres d'art comme « une sorte de jeu raffiné, une jouissance de luxe, sa distinction particulière reposant dans son divorce absolu de l'utilité » (41). Restée insulaire, cette théorie n'a été connue sur le Continent que comme « esthétique anglaise » (41), raillent les trois auteurs, avant de renvoyer en note à certains de ses épigones dont ils désignent les inconséquences et les apories. Il n'est pas étonnant que ce chapitre sarcastique s'achève par le « Don Quichotte » de Goya, image ironique des Victoriens partisans des grands systèmes explicatifs et de la philosophie synthétique.

Un haut relief d'Amarāvatī du $\mathrm{II}^{\mathrm{e}}$ siècle (reproduction $\mathrm{H}$ ) représentant des femmes et des hommes auprès d'un cheval introduit le chapitre "Le plaisir ». Toutefois, la théorie d'une beauté comme plaisir objectifié soutenue par G. Santayana confondant la beauté et le plaisir réduit le lexique esthétique et échoue à décrire la valeur des œuvres d'art (45). Ce sont encore les poètes qui l'expriment le mieux puisque le « Sonnet composé sur la plage près de Calais » de Wordsworth clôt le chapitre : " God being with thee when we know it not. » (46) Sans langage précis, il y a fort à parier que nous resterions dans la position ignorante de ce «nous » évoqué par le poète romantique. 
Quelles émotions l'art peut-il ou doit-il susciter ? Quelle en est la valeur ? Cette valeur doit-elle être évaluée seulement comme émotion ? Le chapitre XI examine ainsi « les prétentions de l'émotionaliste » (47, on appréciera le néologisme) soutenant l'origine émotionnelle de toutes les œuvres [...] ce qui revient à confondre l'art avec des productions inférieures, sarcastiquement listées : la poésie de guerre, celles des collégiennes et une bonne part de la poésie religieuse (47). L'art peut certes susciter des émotions valables, comme en témoigne (ironiquement ?) le poème «Coronach » de W. Scott (47) ${ }^{23}$, mais il ne s'ensuit nullement que l'on réponde à la question : pourquoi l'émotion est-elle désirable?

Quant à la tentative de certains esthéticiens de restreindre leur champ à une « unique émotion » (48), qu'elle soit collective ou individuelle, elle a été jugée trop vague par les psychologues qui n'ont pu lui attribuer de valeur précise. L'émotion peut faire l'objet d'une expérience dépourvue de signification particulière et recevoir une place importante sans pour autant relever des préoccupations de l'art (48). Goya clôt cette méditation avec la gravure « Une victime de l'Inquisition » dont la valeur d'émotion est indéniable, mais que l'artiste a montré sous des aspects quasi caricaturaux puisque le personnage principal, affublé d'un grand chapeau pointu, baisse le nez (reproduction K).

Il est significatif que le chapitre XII, « La forme signifiante ", traite des théories de C. Bell et R. Fry et qu'il se double d'une longue note défendant la nécessité de la précision langagière dans l'appréciation esthétique contre les tenants de la seule expérience, ineffable de surcroît. ${ }^{24}$ Il est également significatif que ce chapitre soit dépourvu d'illustration et se conclue par les trois derniers vers de « Lo, Victress on the Peaks » de Whitman, court poème des Feuilles d'herbe remettant en cause toute liberté illimitée, et symptomatiquement raccourci pour célébrer sa limite : le trépas et le verbe (53). Précisant leur exigence d'exactitude langagière, les auteurs soulignent la nécessité de distinguer « l'utilisation évocatrice du langage et son utilisation scientifique » $(53)^{25}$.

L'adoration du Bouddha d'Amarāvatī (reproduction L) ouvre le chapitre XIII, « L'empathie »,

23. Le coronach est un chant funèbre improvisé d'origine irlandaise ou écossaise.

24 Les auteurs ont emprunté à C. Bell le titre de son article de 1913, « Art and Significant Form ». La " forme signifiante » dérive des écrits de W. Pater et elle occupe une place importante pour le groupe de Bloomsbury. Richards a quant à lui également été un lecteur attentif de Pater. Voir John Paul Russo, I. A. Richards. His Life and Work, Baltimore: The Johns Hopkins Press, 1989, p. 20-30.

25 En introduction, les auteurs avaient souligné : «We assume too readily that similar language involves similar thoughts and similar things thought of. » (9) 
où les auteurs traitent de l'Einfülhung formulé par Theodor Lipps, Hermann Lotze ${ }^{26}$ et Vernon Lee. La description de notre émotion peut donner lieu à un récit de l'organisation de nos impulsions à un moment donné et par rapport à nos expériences antérieures, ou à « une explication en termes physiopsychologiques de l'art en termes de stimulus pour en décrire ses effets sensoriels immédiats et les impulsions que ces derniers mettent en jeu » (55). Dans les deux cas, il s'agit d'un " ensemble très compliqué de conditions » qu'illustre humoristiquement la plainte du roi insomniaque d'Henry IV, Part II (III, 1) de Shakespeare (55-6).

Qu'il s'agisse des esthéticiens ou du critique d'art B. Berenson cité en note, tous échouent à formuler « une théorie esthétique complète » parce qu'aucun ne peut répondre à la question : "Qu'estce que la beauté ?»(58). Résumant le débat dans Beauty and Ugliness, V. Lee aboutit à des conclusions très confuses (58). L'empathie a la faiblesse d'une théorie du plaisir " énoncée sous une forme plus vague et une perspective plus limitée », puisqu'elle ne permet pas de différencier une promenade à bicyclette et l'art (58). L'exemple est caustique parce qu'il renvoie aux cyclistes, principalement de sexe féminin, des années 1890, mais aussi aux expériences très concrètes qu'a analysées Lee. Raillant l'« empathiste enthousiaste pur sang » (59) toujours capable de découvrir un champ appelé " esthétique », les auteurs le laissent in fine à sa bonne mort ${ }^{27}$. L'Einfülhung n'est pas une théorie esthétique cohérente, mais elle permet de s'approcher du bon « champ esthétique », peut-être désigné par une tête de jeune femme du Bénin du XVI siècle (reproduction M) illustrant l'une des propositions de V. Lee : seuls les objets suscitant notre plaisir en facilitant notre vitalité peuvent être dits beaux.

\section{Synesthésie?}

Le chapitre XIV, "La synesthésie ", est le plus long, et se voit illustré par de nombreux extraits poétiques - le Prélude de Wordsworth (62), la poésie de Tennyson (63), de Milton (66), Jammes, ThonSin-Yu (70), R. Bridges (73) - et par la prose de Tolstoï (67). Il s'orne également de la photogravure d'une houle (reproduction $\mathrm{A}$ ), obligeant en quelque sorte le lecteur docile ou curieux à retourner au début de l'ouvrage où il redécouvrira l'idéogramme et la citation du Zhong Yong. Les trois premiers

26 H. Lotze, Mikrokosmos. Ideen zur Naturgeschichte und Geschichte der Menschheit (1856). Voir Gerald Hartung, " Le Mikrokosmos de Hermann Lotze et le discours anthropologique en Allemagne au XIX ${ }^{\mathrm{e}}$ siècle », Revue germanique internationale, 2009, $\mathrm{n}^{\circ} 10 . \mathrm{http} / /$ rgi.revues.org/323

27 « it is hardly worth while to set up and overturn dummies for hypothetical monomaniacs. » (59) 
quatrains de « Break, break, break » de Tennyson (63), scandent habilement ${ }^{28}$ la systématisation des impulsions auxquelles correspondent des émotions simples et des attitudes, comme le fait la reproduction correspondant ici à « l'expérience de la beauté » (64). Immobile et pourtant en mouvement, la mer illustre la passivité active du sujet faisant l'expérience de la synesthésie, en s'impersonnalisant, et en accédant au résultat d'un processus similaire chez le créateur. Elle est également la représentation ou l'évocation d'un espace aux contours flous, indéterminés, où s'effectue peut-être sa rencontre avec le créateur. Cette impersonnalisation, illustrée par cinq vers du « Lycidas » évoquant de nouveau le trépas (66), permet la perception de la mise en relation à d'autres impulsions, tandis qu'une note cite Guerre et paix de Tolstoï à titre d'illustration (67).

La mer est le lieu atopique de la relation esthétique, au début du processus interprétatif de l'art, lorsque s'opère la rencontre avec la personnalité de l'artiste et que se pose la question de ce qu'il a mis de lui-même dans son œuvre. Le contemplateur est alors invité à faire l'expérience d' « une sélection » et d' " un arrangement » ponctuels des impulsions de l'artiste, à travers les siennes (63). Sauf à utiliser la télépathie, l'œuvre est notre seule façon d'entrer en contact avec d'autres esprits. Lorsque nos impulsions se systématisent et s'intensifient tout en préservant leur libre jeu (l'arnoldien «free play») sans frustration, nous faisons alors l'expérience de beauté, comme l'illustre le Frontispice auquel le lecteur est invité à retourner (64).

Cet état d'équilibre n'est pas passif ; une note cite l'essayiste français C. Lalo tout en renvoyant à la photogravure de la houle (reproduction A), faisant finalement confluer corps du texte, notes et image. Cet état où les impulsions sont expérimentées «ensemble » - l'harmonie - doit bien être qualifié de synesthésie (64) car il est à la fois équilibre et harmonie des impulsions. C'est peut-être celui du lecteur de The Foundations, s'il est prêt à lire deux longues notes, la première fournissant le cadre historique à la thèse développée en renvoyant à Colour-Harmony de Wood ${ }^{29}$ pour l'opposer aux dualistes que sont Kant, Schiller, Platon ; la seconde reconnaissant le caractère spéculatif de la théorie synesthétique à travers l'opposition du psychologue Münsterberg et de l'artiste Cézanne (64).

La partie d'un rouleau de soie montrant les «Admonitions de l'instructrice au Palais » par $\mathrm{Ku}$ K'ai-chih (Chine IV siècle, reproduction $\mathrm{N}$ ) possède également une valeur concordante au texte puisque l'expérience de la beauté abolit non l'altérité, mais ce qu'il faut bien appeler l'exotisation des hommes et des choses : «Le gouffre qui nous sépare des peuples anciens, des sauvages, des ennemis,

28 Le quatrième déplore le jour qui ne reviendra plus.

29 J. Wood, Colour-Harmony, London: Kegan Paul, 1922. 
des gens d'un autre sexe, des enfants, des personnes âgées » (67). Les anthropologues qui auraient dû trouver la valeur de leurs propres recherches en étudiant la valeur ainsi reconnue à l'art ne méritentils pas d'être ironiquement tancés ? Ne sont-ils pas responsables de cette exotisation contre laquelle Richards s'inscrira lorsqu'il partira en Chine en 1929 pour enseigner la traduction ${ }^{30}$ ? La question n'est pas simplement scientifique, elle est également éthique. La valeur de l'art non-occidental est celle d'un art exotisé par les Occidentaux, en premier lieu par leur appellation même d'art oriental, et l'on remarquera qu'elle est inexistante dans The Foundations mentionnant des « œuvres d'art du plus haut degré » (81) sans hiérarchiser leur provenance, en les différenciant simplement.

La valeur de l'expérience synesthétique est double : éthique, elle est aussi éducative, et elle le restera toujours pour Richards. Et l'art, le champ esthétique, y apportent une contribution indispensable puisque leur valeur provient de leur pouvoir de créer des différences (3), et de comprendre d'autres personnalités, comme le montrent savoureusement ces «Admonitions » (reproduction N) à laquelle le lecteur est explicitement renvoyé tandis qu'il lira un poème en prose de Francis Jammes en français (69). Certes, l'art n'est pas le seul à susciter une telle intensification, un tel élargissement, mais qu'il le fasse lui donne sa valeur.

Et si l'on cherche par ailleurs à comprendre comment le divorce entre l'art et d'autres champs a pu se développer en même temps que ses vertus étaient indûment louées ${ }^{31}$, il convient de reprendre l'histoire de l'esthétique, soit l'histoire des théories dites « intellectualistes » (69), marquées par Kant, théoricien de la raison et de l'entendement, et par Schiller pour qui la beauté naît de la tension des deux impulsions humaines que sont le sens et la forme. Or leurs termes sont trop ambigus pour nos auteurs. La XXII des Lettres sur l'éducation esthétique de l'homme fait de la jouissance de la beauté le résultat d'un état d'équanimité et de liberté spirituels unis à la capacité d'activité ${ }^{32}$, jouissance signant l'atteinte de l'état esthétique. Relue à travers le prisme de la synesthésie, elle permet d'opposer l'équilibre

30 Voir son Mencius on the Mind: Experiments in Multiple Definition, London: Kegan Paul, Trench, Trubner \& Co.; New York: Harcourt, Brace, 1932.

31 Cette réflexion de 1920 fait étrangement écho aux thèses de Jacques Bouveresse sur l'époque actuelle où la littérature se voit attribuer un rôle recteur de la philosophie, - « la tendance caractéristique de certains courants postmodernes, à ériger la littérature en une sorte de genre suprême, dont la philosophie et la science elles-mêmes ne sont au fond que des espèces » - ce qui a pour effet de nier leurs spécificités au regard d'une politique qui l'ignore totalement. Voir, La Connaissance de l'écrivain. Sur la littérature, la vérité et la vie, Marseille : Agone, 2008, p. 29

32 Dans cette lettre, Schiller soutient que l'expérience de la beauté ne possède aucune utilité, mais permet à un individu de se créer et lui restitue sa liberté. 
exemplaire de «A Passer By » de R. Bridges (73-4), au quatrain initial de « Thou careless, Awake! » du même poète, cité en note et exemple d' " un équilibre formel sans synesthésie », c'est-à-dire sans harmonisation des impulsions (74). Une note précise en outre qu'il ne faut pas confondre santé et vitalité avec l'art, en renvoyant à la reproduction d'un étonnant chat en bronze (reproduction O) (74). Ce chat égyptien, dont seul apparaît la moitié du corps tendu, permet de distinguer la santé physique et mentale de l'équilibre sur lequel repose la synesthésie - équilibre des impulsions empêchant leur tendance fondamentale à se transformer en action (76) - pour éviter toute confusion entre " l'Atelier et le Gymnase » (74). Car qui fait cette confusion se retrouve, sinon moitié de chat, du moins, moitié de sujet ! Énigmatique au premier regard, cette œuvre somme toute humoristique conclut une série d'illustrations visuelles dont la valeur au regard du texte a été très souvent empreinte d'humour et de décalage. À l'image échoit la captatio benevolentia du lecteur tandis que l'ironie du texte affirme la distance prise avec les théories insatisfaisantes de la beauté et leurs partisans tout en se voyant renforcée par les citations littéraires.

Il revient au Song Yong de clore l'ouvrage, inscrivant l'ultime touche d'altérité qui l'a caractérisé, tandis que les auteurs affirment que « la valeur finale de l'équilibre est qu'il vaut mieux être totalement en vie que partiellement. » (77) La "Conclusion » souligne que l'équilibre constitue une justification pour « la préférence d'une expérience sur une autre » puisque l'expérience synesthétique «met en jeu toutes nos facultés » (76), et qu'elle nous permet d'apprécier certaines relations entre divers éléments différemment des circonstances habituelles. L'équilibre constitue sans doute cette « éducation esthétique » pensée entre autres par Schiller et Walter Pater, et qui trouve son plein développement chez les Cambridgiens.

Tant le(s) texte(s) que l'image ont déstabilisé toute habitude de lecture, et ont cherché à transformer le lecteur lambda en exégète, puis à le mettre au travail. La synesthésie est donc à comprendre comme une théorie dont ses auteurs revendiquent le caractère étranger, mais non exotique, et novateur au regard des grands systèmes interprétatifs victoriens dépassés. Elle est également un processus de dépersonnalisation/repersonnalisation à travers la médiation du différent. Dans ce processus, l'illustration perd sa définition classique pour devenir stimulus physico-psychologique abolissant la distinction du texte et de l'image, du langage critique et de la littérature. Ce n'est pas le moindre intérêt de cette esthétique moderniste qui cherche à contrer les thèses des tenants officiels du Modernisme que sont Bell et Fry pour des raisons éthiques et politiques.

The Foundations of Aesthetics pose en outre les jalons conceptuels de la réflexion poétique de Richards dans les années 1920. Rédigé au moment où se crée la faculté d'anglais de Cambridge dont il 
fera partie, il apparaît comme une formulation de son esthétique en établissant les domaines sur lesquels se fonderont ses analyses textuelles : la psychologie, dont il fut l'étudiant passionné, et l'analyse du langage. Définie comme la «théorie de la Beauté par excellence »(80-1), la synesthésie annonce la définition de la valeur poétique comme harmonisation d' " impulsions disparates » présentée dans ses ouvrages suivants : The Meaning of Meaning (avec C. K. Ogden, 1923) ; The Principles of Literary Criticism (1925) ; Poetries and Sciences (1926) ; Practical Criticism: A Study of Literary Judgement (1929). Derrière son ironie et son humour très éloignés du cliché du ponte d'Harvard, Richards apparaît là comme un critique clé du $\mathrm{XX}^{\mathrm{e}}$ siècle, revendiquant la valeur de l'art et tentant de définir un « champ esthétique » qui ne soit pas coupé du champ politique ${ }^{33}$.

33 Voir l'excellente synthèse de G. A. Rudolph, « The Aesthetic Field of I. A. Richards », The Journal of Aesthetics and Art Criticism, 14. 3 (Mar., 1956), p. 348-358. 


\section{Ouvrages Cités}

ARNOLD, Matthew. "The Function of Criticism at the Present Times ». 1864. Lectures and Essays in Criticism. Edited by R. H. Super. Ann Arbor: University of Michigan Press, 1962.

BOUVERESSE, Jacques. La Connaissance de l'écrivain. Sur la littérature, la vérité et la vie. Marseille : Agone, 2008.

HARTUNG, Gerald. «Le Mikrokosmos de Hermann Lotze et le discours anthropologique en Allemagne au XIX siècle ». Revue germanique internationale, $\mathrm{n}^{\circ} 10$ (2009). http://rgi.revues.org/323

KAVALOSKI, Joshua. «The New Critics and the Social Function of Modern Literature ». High Modernism: Aestheticism and Performativity in Literature of the 1920's. London: Camden House, Inc, 2014. 39-72.

KOHLMANN, Benjamin. " Responsible Propagandists: I. A. Richards, T. S. Eliot and Cambridge Experiment ». Committed Styles: Modernism, Politics, and Left-Wing Literature in the 1930s. Oxford: OUP, 2014. 18-53.

OGDEN, Charles K., Ivor Armstrong RICHARDS, and James WOOD. The Foundations of Aesthetics. 1925. Ed. John Constable. London and New York: Routledge, 2001.

The Selected Letters of I. A. Richards. Ed. J. Constable. Oxford: Clarendon, 1990. «The Sense of Beauty », Cambridge Magazine, 10.2 (Jan-Mar. 1921): 73-93.

PATER, Walter. «Preface ». The Renaissance. Studies in Art and Poetry. 1873. Ed. Adam Philips. Oxford: Oxford Classics, 1988.

RICHARDS, I. A. The Principles of Literary Criticism. 1924. London: Routledge, 1989. Science and Poetry. 1926. London: Kegan Paul, Trench, Trubner, 1926.

Mencius on the Mind: Experiments in Multiple Definition. London: Kegan Paul, Trench, Trubner \& Co.; New York: Harcourt, Brace, 1932.

RUSSO John Paul. I. A. Richards. His Life and Work, Baltimore: The Johns Hopkins Press, 1989.

VALENTINE, C. W. « Review of The Foundations of Aesthetics », Mind, ns, 32.125 (Jan., 1923): 120.

WOOD, James. Colour-Harmony. London: Kegan Paul, 1922.

\section{LiSTE DES ILLUSTRATIONS}

Fig. 1 Planche II et page 23 de OGDEN, C. K., I. A. RICHARDS, and James WOOD. The Foundations of Aesthetics. London: Allen, 1925.

Fig. 2 Planche I et page 13 de OGDEN, C. K., I. A. RICHARDS, and James WOOD. The Foundations of Aesthetics. London: Allen, 1925. 